\title{
Dose-response to different radiochemotherapy regimens in locally advanced pancreatic cancer
}

\author{
B.C. Ferreira ${ }^{1,2,3}$, J. Dias ${ }^{2,4}$ A. Gomes ${ }^{1}$, P. Mavroidis ${ }^{5,6}$ and H Rocha ${ }^{2,4}$ \\ ${ }^{1}$ School of Health, Polytechnic of Porto, Porto, Portugal \\ ${ }^{2}$ Institute for Systems Engineering and Computers at Coimbra, Coimbra University, Coimbra, \\ Portugal \\ ${ }^{3}$ I3N, Physics Department of Aveiro University, Aveiro, Portugal \\ ${ }^{4}$ CeBER and FEUC, Coimbra University, Coimbra Portugal \\ ${ }^{5}$ Department of Radiation Oncology, University of North Carolina at Chapel Hill, NC, USA \\ ${ }^{6}$ Division of Medical Radiation Physics, Karolinska Institutet and Stockholm University, \\ Stockholm, Sweden \\ bcf@ess.ipp.pt
}

\begin{abstract}
Conformal radiation therapy (RT) delivered concomitantly with chemotherapy including 5-fluorouracil (5-FU) or Gemcitabine (GEM) is a common treatment for patients with unresectable locally advanced pancreatic tumors. In this study, the Poisson model describing tumor response to these two treatment options was derived. Clinical data was retrieved from reports published from 1990 to 2015. Dosimetric and clinical data from 1196 patients treated with RT with concurrent 5-FU or GEM were gathered. RT doses ranging from 3.6-64.8 Gy, delivered in fractions of 1.2-8 Gy, were converted to a 2 Gy fractionation scheme using the Biological Effective Dose concept. The parameters of the Poisson-Linear-Quadratic-Time model were derived using genetic algorithm optimization to minimize the least-square fitting error. The goodness of the fit was assessed using the Pearson $\chi^{2}$-test. For RT+5-FU, $D_{50}$ was 56.5 Gy, $\gamma$ was $3, \alpha / \beta$ was $4.4, T_{\text {pot }}$ was 36 days and Tk was 23 days. For RT+GEM, $D_{50}$ was $48.3 \mathrm{~Gy}$, $\gamma$ was $3, \alpha / \beta$ was $6.4, T_{\text {pot }}$ was 36 days and Tk was 23 days. As expected, RT+GEM showed higher efficacy than RT+5-FU. A RT dose-response effect was obtained showing that treatment strategies allowing a dose-escalation in pancreas tumors should be investigated.
\end{abstract}

Keywords: Pancreas Tumors, Dose-Response Models, Radiation Therapy, Chemotherapy.

\section{Introduction}

Pancreatic tumors are amongst the most challenging cancer types. Patients are generally diagnosed with the disease in advanced stages for which surgery is no longer viable. Different chemotherapy regimens delivered alone or in combination with RT, have largely been used without achieving long-term overall success. Survival rates are thus 
generally poor and consensus about the best treatment option for each patient has not been established [1].

In concomitant radiochemotherapy, the most common chemotherapy agents are 5fluorouracil (5-FU) and gemcitabine (GEM). These have frequently been used as single agents but have also been combined with different regimens [2-34]. Better overall survival and progression-free survival was generally obtained with radiochemotherapy based on the GEM agent compared to the 5-FU [35,36]. Interestingly in a network metaanalysis that compared different GEM regimens, the combination of RT with GEM has shown to be the most effective GEM based treatment compared to the combination of GEM with other chemotherapy drugs [37]. With conformal RT, dose-prescription were mostly confined to values between 50.4-59.4 Gy. A dose escalation appears to be beneficial in terms of local control, progression-free survival and overall survival $[27,33,35,38]$, but limited by the tolerance of surrounding organs at risk. An accurate model describing tumor response to delivered treatments is thus very useful as it may help investigating new treatment approaches for pancreatic cancer.

The aim of this study was to derive the dose-response parameters for the PoissonLinear-Quadratic-Time model for locally advanced pancreatic tumors for patients that received conformal RT concomitant with 5-FU or GEM using the clinical data reported in the literature.

\section{$2 \quad$ Material and methods}

Clinical information was retrieved from scientific papers reporting treatment outcome of patients with locally advanced pancreatic cancer (cases with distant metastasis were excluded). Primary treatment was 3D conformal RT concurrent with 5-FU or GEM and chemotherapy alone (simulating zero RT dose). No prior treatments were delivered to these patients. Thirty three papers, which were published from 1990 to 2015 , with comprehensive descriptions on patients, delivered treatment (chemotherapy regimen, RT prescription dose, fractionation) and response to therapy (according to WHO or RECIST guidelines [39,40]) were included in this study [2-34].

The groups RT+5-FU and RT+GEM were composed by a total of 1196 patients treated from about 1988 to 2008 . RT doses, ranging from 3.6-64.8 Gy delivered in fractions of 1.2-8 Gy in 2-54 fractions, were converted to a common 2 Gy fractionation scheme using the Biological Effective Dose (BED) concept [41]. The best estimates for the delivered doses were made based on the information described in each report about compliance to treatment. Response Rate (RR), as quantified by the sum of the rate of complete and partial tumor response to therapy, was used as the endpoint of interest for the derivation of the dose-response curve. For pancreatic tumors, this endpoint follows more closely the typical sigmoidal shape of radiobiological models than the endpoint local control (given by the sum of RR and the rate of stable disease) [42].

The probability of tumor response to the delivered treatment, $P$, was determined assuming an additive effect between chemotherapy and RT using the expression,

$$
P=P_{c h}+P_{r t}\left(1-P_{c h}\right)
$$


where $P_{c h}$ and $P_{r t}$ are the probability of response to the chemotherapy regimen and the delivered RT, respectively [43]. The probability of response to chemotherapy was calculated as the weighted mean of the RR obtained to chemotherapy delivered as monotherapy [28-31]. The Poisson-Linear-Quadratic-Time model was used to describe tumor response to RT for the groups of patients treated with RT concomitant with GEM $(\mathrm{RT}+\mathrm{GEM})$ or RT concomitant with 5-FU (RT+5-FU). The parameters of this model are: $D_{50}$, the dose that results in a $50 \%$ response; $\gamma$, the maximum normalized doseresponse gradient; $\alpha / \beta$, the fractionation sensitivity parameter of the Linear-quadratic model, $T_{\mathrm{pot}}$, the tumor potential doubling time and $T_{\mathrm{k}}$, the time at which repopulation begins. The parameters: $\alpha, \beta, \gamma, T_{\mathrm{pot}}$ and $T_{\mathrm{k}}$ were derived using genetic algorithm optimization to minimize the least-square fitting error and $D_{50}$ and $\alpha / \beta$ were calculated using the expressions described in Ferreira et al [41]. The goodness of the fit was evaluated using the Pearson $\chi^{2}$-test.

\section{$3 \quad$ Results}

The local control rates of the patient cohorts that received RT+5-FU varied between $25 \%$ and $100 \%$, whereas for those treated with RT+GEM it varied between $33 \%$ and $100 \%$. The RR of the group RT $+5-\mathrm{FU}$ varied between $5 \%$ and $45 \%$, whereas for the $\mathrm{RT}+\mathrm{GEM}$ group it ranged from $5 \%$ to $50 \%$. The average of RR for patients treated with chemotherapy alone with 5-FU was 7.1\% and with GEM was 9\% (range: $4 \%-15 \%$ ). The RR as a function of the delivered dose for a 2 Gy fractionation schedule (EQD2) is shown Fig. 1. The values of the dose-response parameters of the Poisson-LinearQuadratic-Time model are shown in Table 1.

Table 1. Dose-response parameters for the Poisson-Linear-Quadratic Model

\begin{tabular}{llllllll}
\hline Treatment & $\boldsymbol{D}_{\mathbf{5 0}} / \mathbf{G y}$ & $\boldsymbol{\gamma}$ & $\boldsymbol{\alpha} \boldsymbol{\beta} / \mathbf{G y}$ & $\boldsymbol{\alpha} / \mathbf{G y}^{-1}$ & $\boldsymbol{\beta} / \mathbf{G y}^{-2}$ & $\boldsymbol{T}_{\text {pot }} /$ days & $\boldsymbol{T}_{\mathbf{k}} / \mathbf{d a y s}$ \\
\hline RT+5-FU & 56.5 & 3.0 & 4.4 & 0.103 & 0.024 & 36.0 & 23.3 \\
RT+GEM & 48.3 & 3.0 & 6.4 & 0.134 & 0.021 & 36.0 & 23.3 \\
\hline
\end{tabular}

\section{Discussion}

Dose-response curves for pancreatic tumors in function of BED values were first derived by Moraru et al [42] that proposed a biophysical model to describe RR to radiochemotherapy. Durante et al [43] derived dose-response models for different treatment modalities but have selected as endpoint overall survival. In this study, the PoissonLinear-Quadratic-Time model describing RR in function of EQD2 was selected instead.

A smaller $D_{50}$ value for concurrent radiochemotherapy with GEM compared with the $D_{50}$ value obtained for $\mathrm{RT}+5-\mathrm{FU}$ is consistent with the studies showing the higher efficacy of GEM compared to 5-FU [35,36]. During optimization the parameters $\alpha$ and $\beta$ were derived, resulting in a $\alpha / \beta$ smaller than commonly used [42], but for RT+GEM 
similar to the reported by Chapman et al [44]. A low value of $\alpha / \beta$ would explain the good local tumor response obtained with hypofractionation schedules used with Stereotactic Body RT [45].

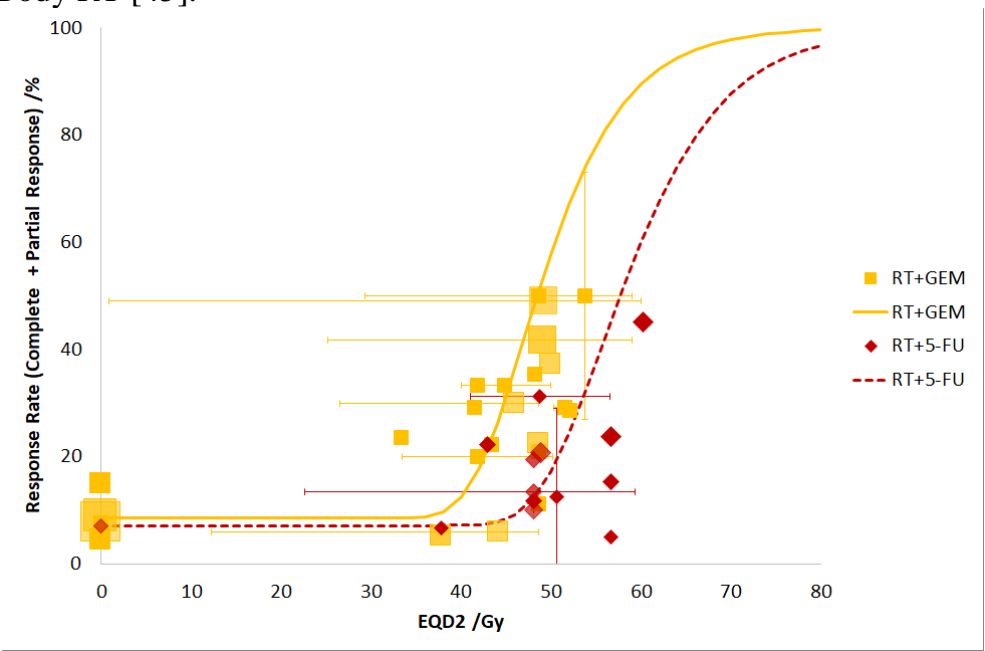

Fig. 1. Dose-response for unresectable locally advanced pancreatic tumors treated with concomitant radiochemotherapy with 5-FU or GEM. Dose-bars show the range of delivered doses and response-bars show its 95\% confidence intervals reported by original authors [2-33].

Ideally, for the accurate derivation of dose-response curves, dosimetric data consisting of the delivered dose, corrected for the delivered fractions and the real overall treatment time, should be used for those patients that achieved complete or partial response. Unfortunately, discrimination between the dose delivered to patients that responded or not to the therapy was never made. Furthermore, although in each study dose prescription was uniform, the delivered dose varied due to the lack of compliance to therapy. Most authors reported a compliance to planned RT of approximately $80 \%$ but without reporting the delay in RT for each patient $[2,7,23,25]$. Thus, corrections for the real overall treatment time were not made. Ideally, the 3-dimensional dose-distribution should be used to assess the dose delivered to the target volume. However, with conformal RT patients with pancreatic tumors are generally irradiated with the box technique for which a good dose homogeneity in the planning target volume is obtained. Despite the uncertainties characterizing the input data, certain reasonable approximations had to be made. The result is a good model fitting as indicated by the good correlation between the dose-response curves and their association with the clinical doseresponse points (Fig. 1).

\section{Conclusion}

The parameters for the Poisson model describing tumor response to radiochemotherapy concomitant with the 5-FU and GEM agents were derived. The determined dose-response curves indicate a good correlation between applied treatments and outcome. The 
generation of large databases integrating the data of all treated patients is largely needed for the development of accurate models describing tumors, and normal tissues, to delivered therapies.

\section{Acknowledgements}

This work was partially funded by project grants UID/CTM/50025/2019, POCI-010145-FEDER-028030 and by the Fundação para a Ciência e a Tecnologia under project grant UID/Multi/00308/2019.

\section{Conflicts of interest}

None.

\section{References}

1. Torgeson A, Tao R, Garrido-Laguna I et al: Large database utilization in health outcomes research in pancreatic cancer: an update. J Gastrointest Oncol. 9(6):996-1004 (2018).

2. Fisher, B.J., Perera, F.E., Kocha, W., et al: Analysis of the clinical benefit of 5-fluorouracil and radiation treatment in locally advanced pancreatic cancer. Int J Radiat Oncol Biol Phys 45(2), 291-5 (1999).

3. Boz, G., Paoli, A., Innocente, R., et al: Radiotherapy and continuous infusion 5-fluorouracil in patients with nonresectable pancreatic carcinoma. Int J Rad Oncol Biol Phys 51(3), 736 740 (2001).

4. Osti, M.F., Costa, A.M., Bianciardi, F. et al: Concomitant radiotherapy with protracted 5fluorouracil infusion in locally advanced carcinoma of the pancreas: a phase II study. Tumori 87(6):398-401 (2001).

5. Morganti, A.G., Forni, F., Macchia, G., et al: Chemoradiation of unresectable pancreatic carcinoma: impact of pretreatment hemoglobin level on patterns of failure. Strahlenther Onkol 179(2), 87-92 (2003).

6. Morganti, A., Valentini, V., Macchia, G., et al: 5-fluorouracil - based chemoradiation in unresectable pancreatic carcinoma: phase I-II dose-escalation study. Int J Radiat Oncol Biol Phys 59(5), 1454-1460 (2004).

7. Ishii H, Okada $\mathrm{S}$, Tokuuye $\mathrm{K}$ et al: Protracted 5-fluorouracil infusion with concurrent radiotherapy as a treatment for locally advanced pancreatic carcinoma. Cancer 79(8),1516-20 (1997).

8. Shinchi, H., Takao, S., Noma, H., et al: Length and quality of survival after external-beam radiotherapy with concurrent continuous 5 -fluorouracil infusion for locally unresectable pancreatic cancer. Int J Radiat Oncol Biol Phys 53(1), 146-50 (2002).

9. Li, C.P., Chao, Y., Chi, K.H. et al: Concurrent chemoradiotherapy treatment of locally advanced pancreatic cancer: gemcitabine versus 5-fluorouracil, a randomized controlled study. Int J Radiat Oncol Biol Phys 57(1):98-104 (2003).

10. Ueno, H., Okusaka, T., Ikeda, M. et al: Phase I study of hyperfractionated radiation therapy with protracted 5-fluorouracil infusion in patients with locally advanced pancreatic cancer. Oncology 67(3-4):215-21 (2004). 
11. Wilkowski R, Boeck S, Ostermaier S et al: Chemoradiotherapy with concurrent gemcitabine and cisplatin with or without sequential chemotherapy with gemcitabine/cisplatin vs chemoradiotherapy with concurrent 5-fluorouracil in patients with locally advanced pancreatic cancer-a multi-centre randomised phase II study. Br J Cancer. 101(11):1853-9 (2009).

12. Pipas, J.M., Mitchell, S.E., Barth, R.J. Jr et al: Phase I study of twice-weekly gemcitabine and concomitant external-beam radiotherapy in patients with adenocarcinoma of the pancreas. Int J Radiat Oncol Biol Phys 50(5):1317-22 (2001).

13. Wolff, R.A., Evans, D.B., Gravel, D.M., et al: Phase I trial of gemcitabine combined with radiation for the treatment of locally advanced pancreatic adenocarcinoma. Clin Cancer Res 7(8):2246-53 (2001).

14. Lange, S.M., van Groeningen, C.J., Meijer, O.W. et al: Gemcitabine-radiotherapy in patients with locally advanced pancreatic cancer. Eur J Cancer 38(9):1212-7 (2002).

15. Okusaka, T., Ito, Y., Ueno, H. et al: Phase II study of radiotherapy combined with gemcitabine for locally advanced pancreatic cancer. Br J Cancer 91(4), 673-7 (2004).

16. Magnino, A., Gatti, M., Massucco, P. et al: Phase II trial of primary radiation therapy and concurrent chemotherapy for patients with locally advanced pancreatic cancer. Oncology 68(4-6), 493-9 (2005).

17. Oya, N., Shibuya, K., Sakamoto, T. et al: Chemoradiotherapy in patients with pancreatic carcinoma: phase-I study with a fixed radiation dose and escalating doses of weekly gemcitabine. Pancreatology 6(1-2), 109-16 (2006).

18. Brade, A., Brierley, J., Oza, A., et al. Concurrent gemcitabine and radiotherapy with and without neoadjuvant gemcitabine for locally advanced unresectable or resected pancreatic cancer: a phase I-II study. Int J Radiat Oncol Biol Phys. 67(4):1027-36 (2007).

19. Igarashi, H., Ito, T., Kawabe, K. et al: Chemoradiotherapy with twice-weekly administration of low-dose gemcitabine for locally advanced pancreatic cancer. World J Gastroenterol. 14(34):5311-5 (2008).

20. Maemura, K., Shinchi, H., Noma, H. et al: Comparison of hyper-fractionated accelerated and standard fractionated radiotherapy with concomitant low-dose gemcitabine for unresectable pancreatic cancer. Anticancer Res 28(4C), 2369-72 (2008).

21. Girard, N., Mornex, F., Bossard, N. et al: Estimating optimal dose of twice-weekly gemcitabine for concurrent chemoradiotherapy in unresectable pancreatic carcinoma: mature results of GEMRT-01 Phase I trial. Int J Radiat Oncol Biol Phys 77(5):1426-32 (2010).

22. Loehrer, P.J. Sr, Feng, Y., Cardenes, H. et al: Gemcitabine alone versus gemcitabine plus radiotherapy in patients with locally advanced pancreatic cancer: an Eastern Cooperative Oncology Group trial. J Clin Oncol 29(31), 4105-12 (2011).

23. Shibuya, K., Oya, N., Fujii, T. et al: Phase II study of radiation therapy combined with weekly low-dose gemcitabine for locally advanced, unresectable pancreatic cancer. Am J Clin Oncol 34(2), 115-9 (2011).

24. Cardenes, H.R., Moore, A.M., Johnson, C.S. et al: A phase II study of gemcitabine in combination with radiation therapy in patients with localized, unresectable, pancreatic cancer: a Hoosier Oncology Group study. Am J Clin Oncol 34(5), 460-5 (2011).

25. Mattiucci, G.C., Morganti, A.G., Valentini, V. et al: External beam radiotherapy plus 24hour continuous infusion of gemcitabine in unresectable pancreatic carcinoma: long-term results of a phase II study. Int J Radiat Oncol Biol Phys 76(3), 831-8 (2010).

26. Small, W. Jr, Berlin, J., Freedman, G.M., et al: Full-dose gemcitabine with concurrent radiation therapy in patients with nonmetastatic pancreatic cancer: a multicenter phase II trial. J Clin Oncol 26(6):942-7 (2008). 
27. Huang, P.I., Chao, Y., Li, C.P. et al: Efficacy and factors affecting outcome of gemcitabine concurrent chemoradiotherapy in patients with locally advanced pancreatic cancer. Int J Radiat Oncol Biol Phys. 73(1), 159-65 (2009).

28. Tada, M., Arizumi, T., Nakai, Y., et al. Efficacy of gemcitabine for locally advanced pancreatic cancer: comparison with 5-fluorouracil-based chemoradiotherapy. Chemotherapy. 54(4):302-8 (2008).

29. Yanagimoto, H., Ishii, H., Nakai, Y., et al. Improved survival with combined gemcitabine and S-1 for locally advanced pancreatic cancer: pooled analysis of three randomized studies. J Hepatobiliary Pancreat Sci. 21(10):761-6 (2014).

30. Louvet, C., Labianca, R., Hammel, P. et al Gemcitabine in combination with oxaliplatin compared with gemcitabine alone in locally advanced or metastatic pancreatic cancer: results of a GERCOR and GISCAD phase III trial. J Clin Oncol. 23(15):3509-16 (2005).

31. Evans, T.R.J., Van Cutsem, E., Moore, M.J. et al: Phase 2 placebo-controlled, double-blind trial of dasatinib added to gemcitabine for patients with locally-advanced pancreatic cancer. Ann Oncol. 28(2):354-361 (2017).

32. Cengiz, M., Zorlu, F., Yalcin, S. et al: Concurrent gemcitabine and radiotherapy for locally advanced pancreatic cancer. Med Oncol. 24(2):239-43 (2007).

33. Ogawa, K., Ito, Y., Hirokawa, N. et al: Concurrent radiotherapy and gemcitabine for unresectable pancreatic adenocarcinoma: impact of adjuvant chemotherapy on survival. Int J Radiat Oncol Biol Phys. 83(2):559-65 (2012).

34. Cullinan, S., Moertel, C.G., Wieand, H.S. et al: A phase III trial on the therapy of advanced pancreatic carcinoma. Evaluations of the Mallinson regimen and combined 5-fluorouracil, doxorubicin, and cisplatin. Cancer. 65(10):2207-12 (1990).

35. Huang, J., Robertson, J.M., Margolis, J. et al: Long-term results of full-dose gemcitabine with radiation therapy compared to 5-fluorouracil with radiation therapy for locally advanced pancreas cancer. Radiother Oncol 99(2):114-9 (2011).

36. Zhu, C.P., Shi, J., Chen, Y.X. et al: Gemcitabine in the chemoradiotherapy for locally advanced pancreatic cancer: a meta-analysis. Radiother Oncol. 99(2):108-13 (2011).

37. Chen, J., Chen, L., Yu, J. et al: Meta-analysis of current chemotherapy regimens in advanced pancreatic cancer to prolong survival and reduce treatment-associated toxicities. Mol Med Rep. 19(1):477-489 (2019).

38. Tozzi, A., Comito, T., Alongi, F. et al: SBRT in unresectable advanced pancreatic cancer: preliminary results of a mono-institutional experience. Radiat Oncol 8:148 (2013).

39. World Health Organisation. WHO handbook for reporting results of cancer treatment. Offset Pub. 48. Geneva: World Health Organisation, (1979).

40. Therasse, P., Arbuck, S.G., Eisenhauer, E.A., et al. New guidelines to evaluate the response to treatment in solid tumors. European Organization for Research and Treatment of Cancer, National Cancer Institute of the United States, National Cancer Institute of Canada. J Natl Cancer Inst. 92:205-16 (200).

41. Ferreira, B.C., Lopes, M.C., Mateus, J., et al: Radiobiological evaluation of forward and inverse IMRT using different fractionations for head and neck tumours. Radiat. Oncol 5:57 (2010).

42. Moraru, I.C., Tai, A., Erickson, B., and Li X.A.: Radiation dose responses for chemoradiation therapy of pancreatic cancer: an analysis of compiled clinical data using biophysical models. Pract Radiat Oncol 4(1),13-9 (2014).

43. Durante, M., Tommasino, F., Yamada, S.: Modeling Combined Chemotherapy and Particle Therapy for Locally Advanced Pancreatic Cancer. Front Oncol. 5:145 (2015).

44. Chapman, J.D. Can the two mechanisms of tumor cell killing by radiation be exploited for therapeutic gain? J Radiat Res. 55(1):2-9 (2014). 
45. De Bari, B., Porta, L., Mazzola, R. et al. Hypofractionated radiotherapy in pancreatic cancer: Lessons from the past in the era of stereotactic body radiation therapy. Crit Rev Oncol Hematol. 103:49-61 (2016). 